\title{
Numerical Simulation of Flow-Induced Flexoelectric Effect in Liquid Crystals ${ }^{*}$
}

\author{
Takanori NAGAE ${ }^{* *}$, Tomohiro TSUJI ${ }^{* * *}$ and Shigeomi CHONO ${ }^{* * *}$ \\ ${ }^{* *}$ Graduate School, Kochi University of Technology, Tosayamada-cho, Kami-shi, Kochi, 782-8502, Japan \\ ***Department of Intelligent Mechanical Systems Engineering, Kochi University of Technology, \\ Tosayamada-cho, Kami-shi, Kochi, 782-8502, Japan \\ E-mail : chono.shigeomi@kochi-tech.ac.jp
}

\begin{abstract}
As a fundamental research for developing new liquid crystalline devices, numerical simulations on flexoelectric polarization in liquid crystals under shear flow has been performed using the Leslie-Ericksen continuum theory. We have used two types of liquid crystals for computation; one is N-(p-methoxybenzylidene)-p'butylaniline (MBBA), which is an aligning liquid crystal, and another is 4-n-Octyl4'-cyanobiphenyl (8CB), which is a tumbling liquid crystal. These liquid crystals are placed between two parallel plates, and one plate is moved to its plane direction in order to impose shear flow on the liquid crystals. For simplification, the director is assumed to lie in the shear plane. As the boundary condition of the director, the director orientation on one plate is anchored strongly, and the director on the other plate is weakly anchored so that the director orientation depends on shear rate. The flexoelectric polarization for the aligning liquid crystal increases monotonically to reach a steady state value, while it shows peaks for the tumbling liquid crystal; the number of peaks depends on the Ericksen number (ratio of viscous to elastic stress), and the peak value is considerably larger than the steady state value for the aligning liquid crystal. Since the flexoelectric polarization is estimated from the difference of the director angle between both plates, it is found that the flexoelectric polarization can be controlled by the anchoring strength as well as the Ericksen number.
\end{abstract}

Key words: Numerical Analysis, Non-Newtonian Flow, Liquid Crystal, Flexoelectric Effect, Shear Flow, Anchoring Strength

\section{Introduction}

Liquid crystalline materials exhibit optical, electrical, and viscous anisotropies ${ }^{(1)(2)}$. Liquid crystal displays are operated by utilizing the optical anisotropy of the liquid crystals for light switching. Because of their low power consumption and thin shape, the market of the liquid crystal displays rapidly grew in the last decade. Recently, a different industrial application of the liquid crystalline materials has been proposed by Chono et $\mathrm{al}^{(3)(4)}$. They analyzed the liquid crystalline flow induced by electric fields under various anchoring conditions, leading to the fact that the induced flow can be controlled by changing the anchoring conditions. While the total induced flow rate in the liquid crystalline cell is 0 for 0 deg twist anchoring angle, the flow rates are finite values for non- 0 deg twist angles. Using this induced flow, liquid crystalline actuators have been proposed as a new application of liquid crystals. Since there is no restriction on the size and shape, the liquid crystalline actuators can be used in micro-robots, micro-machines, and MEMS.

Since the liquid crystals have novel features which do not appear in usual liquids or 
usual solids, there must be many liquid crystal applications to be discovered. To find further application of the liquid crystals, huge amount of fundamental researches must be performed for the materials.

It is known that the liquid crystalline materials show macroscopic electric polarization, called flexoelectric polarization ${ }^{(5)(6)}$, depending on the spatial configuration of constitutive molecules. Figures 1(a) and (b) are schematics of the mechanism of the flexoelectric polarization. Boxes represent the liquid crystalline molecules and the arrows are the polarization direction. When the liquid crystalline material is placed between two parallel plates and the molecular orientation field is homogeneous, there is no order in the polarization direction and the material is macroscopically unpolarized (Fig.1(a)). On the other hand, if the material is placed between wedge-shaped plates, the distortion in the molecular orientation field arises and the macroscopic flexoelectric polarization is induced through the symmetry breaking of the polarization direction (Fig.1(b)).

Our final goal is to develop new micro-sensing devices, which are able to detect the velocity, posture, position, and force of objects. In comparison with the solid piezoelectric materials, the liquid crystalline materials have the following features; (1) the flexoelectric polarization is sensitive to the rate of strain as well as the strain of objects, (2) they do not have specific shapes and sizes, and (3) the infinite deformation is allowed. To utilize these features for the micro-sensing devices, the relationship between the dynamics of the molecular configurations and the flexoelectric polarization must be clearly understood.

After the discovery of the flexoelectric polarization of the liquid crystals, many fundamental researches have been performed both theoretically and experimentally ${ }^{(7)-(10)}$. Flows also possess the effect of producing the inhomogeneity in the molecular orientation field. Thus, it is expected that the flows are able to induce the macroscopic polarization. While many researches on the flow properties of nematic liquid crystals have been done in the past ${ }^{(11)-(13)}$, few researches on the flow-induced polarization have been performed.

In this paper, we focus our attention on the relationship between the macroscopic flexoelectric polarization and the liquid crystalline flow. Using the Leslie-Ericksen continuum theory ${ }^{(14)-(16)}$, the liquid crystalline shear flows between parallel plates are simulated to estimate the flow-induced macroscopic polarization.

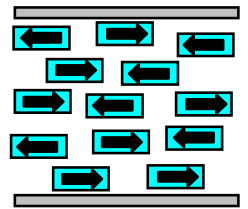

(a)

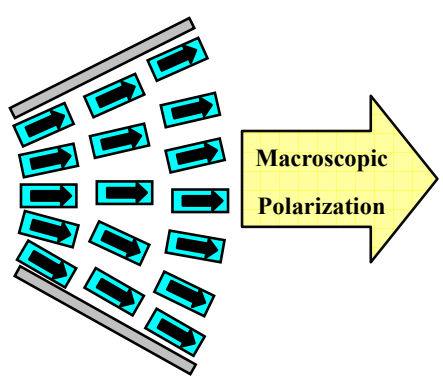

(b)

Fig.1 Molecular model of flexoelectric effect

\section{Governing Equations and Numerical Simulations}

\subsection{Governing Equations}

Governing equations for isothermal incompressible fluids are as follows.

The equation of continuity:

$$
\nabla \cdot \mathbf{v}=0 \text {. }
$$


The equation of motion:

$$
\rho \frac{\mathrm{D} \mathbf{v}}{\mathrm{D} t}=-\nabla p+\nabla \cdot \boldsymbol{\tau}
$$

Here, $\mathbf{v}$ is the velocity vector, $\rho$ the fluid density, $\mathrm{D} / \mathrm{D} t$ the material derivative, and $p$ the pressure. $\tau$ is the extra stress tensor, which is written as follows using the Leslie-Ericksen continuum theory ${ }^{(13)-(15)}$ for nematic liquid crystalline flows,

$$
\boldsymbol{\tau}=\alpha_{1} \mathbf{n n n} \cdot \mathbf{D} \cdot \mathbf{n}+\alpha_{2} \mathbf{n N}+\alpha_{3} \mathbf{N n}+\alpha_{4} \mathbf{D}+\alpha_{5} \mathbf{n n} \cdot \mathbf{D}+\alpha_{6} \mathbf{D} \cdot \mathbf{n n}-\frac{\partial F}{\partial \nabla \mathbf{n}} \cdot(\nabla \mathbf{n})^{T} .
$$

Here, $\alpha_{1}, \ldots, \alpha_{6}$ are the Leslie viscosities, and $\mathbf{n}$ is a unit vector which represents the local averaged orientation direction of rod-like liquid crystalline molecules, called the director. D is the rate-of-strain tensor, and $\mathbf{N}$ the angular velocity vector of the director relative to the background flows, given by:

$$
2 \mathbf{D}=(\nabla \mathbf{v})^{T}+(\nabla \mathbf{v})
$$

and

$$
\mathbf{N}=\frac{D \mathbf{n}}{D t}-\mathbf{\Omega} \cdot \mathbf{n}
$$

The vorticity tensor $\Omega$ is

$$
2 \Omega=(\nabla \mathbf{v})^{T}-(\nabla \mathbf{v}) .
$$

$F$ in Eq.(3) is the Frank free energy density of the director field and is defined as

$$
2 F=K_{1}(\nabla \cdot \mathbf{n})^{2}+K_{2}(\mathbf{n} \cdot \nabla \times \mathbf{n})^{2}+K_{3}|\mathbf{n} \times \nabla \times \mathbf{n}|^{2} .
$$

Here, $K_{1}, K_{2}, K_{3}$ are the Frank elastic constants, corresponding to the splay, twist, and bend deformations of the director field. Since the velocity field has strong interaction with the director field, the director field must be solved simultaneously with the velocity field. The angular momentum equation for the director is

$$
\mathbf{n} \times\left\{\left(\frac{\partial F}{\partial \mathbf{n}}\right)-\nabla \cdot\left(\frac{\partial F}{\partial \nabla \mathbf{n}}\right)+\gamma_{1} \mathbf{N}+\gamma_{2} \mathbf{D} \cdot \mathbf{n}\right\}=\mathbf{0} .
$$

Here, $\gamma_{1}$ and $\gamma_{2}$ are the rotational and irrotational viscosities related to the Leslie viscosities as follows:

$$
\gamma_{1}=\alpha_{3}-\alpha_{2}
$$

and

$$
\gamma_{2}=\alpha_{6}-\alpha_{5}
$$

Once the director field is determined by solving the above governing equations, the macroscopic flexoelectric polarization can be estimated by integrating the following local flexoelectric polarization vector ${ }^{(5)}$, 


$$
\mathbf{P}=e_{11} \mathbf{n}(\nabla \cdot \mathbf{n})+e_{33}(\nabla \times \mathbf{n}) \times \mathbf{n}
$$

Here, $e_{11}$ and $e_{33}$ are the flexoelectric coefficients corresponding to the splay and bend deformations of the director field. It should be noticed that the twist deformation of the director field has no contribution to the flexoelectric polarization.

\subsection{Flow geometry and coordinate systems}

Figure 2 shows the flow geometry and the coordinate systems used in this work. The liquid crystalline materials are placed between parallel plates with the gap $H$. A shear flow is applied to the liquid crystals by moving the upper plate to the $x$-direction with velocity $U$ and thus the mean shear rate is $U / H$. For the simplification of the numerical calculation, the in-plane assumption of the director is employed, which is commonly used for the simulation of liquid crystalline flows. Under the assumption, the director can be expressed with two components as,

$$
\mathbf{n}(y)=\left(n_{x}(y), n_{y}(y), 0\right)=(\cos \phi(y), \sin \phi(y), 0) .
$$

Here, $\phi$ is an angle between the director and the $x$-axis. The velocity vector under the in-plane assumption of the director is,

$$
\mathbf{v}(y)=(u(y), 0,0) .
$$

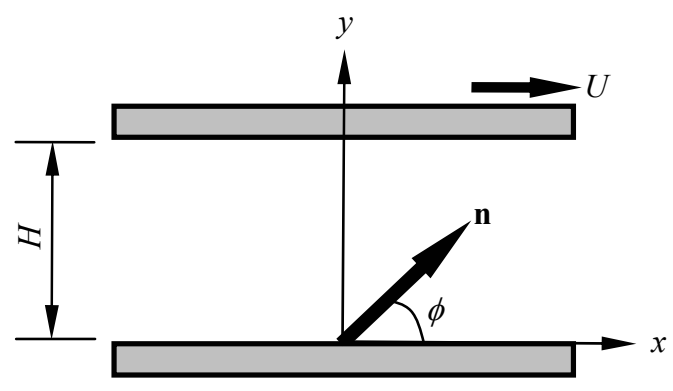

Fig.2 Flow geometry and coordinate systems

\subsection{Numerical simulation}

After applying the coordinate systems to the governing equations, the equations are non-dimensionalized with the gap $H$, the upper plate velocity $U$, the rotational viscosity $\gamma_{1}$, and the average of the three elastic constants $K\left(=\left(K_{1}+K_{2}+K_{3}\right) / 3\right)$. The Ericksen number $\operatorname{Er}\left(=\gamma_{1} H U / K\right)$, which represents the ratio of viscous force to elastic force, appears in the non-dimensionalized governing equations, and is one of the simulation parameters. When the flow geometry and the material constants are fixed, the Ericksen number is proportional to the upper plate velocity. Here after, the variables with the superscript * are non-dimensional.

The numerical methods used in this work are the finite difference method for spatial discritization and the second order Runge-Kutta method for time integration. After the validation of the numerical procedure, the mesh with $\Delta y^{*}=0.01$ and the time step $\Delta t^{*}=10^{-9}$ are employed throughout the computations.

As mentioned above, the flexoelectric polarization stems from the distortion of the director field. However, the macroscopic flexoelectric polarization becomes 0 even if the liquid crystal is locally polarized, when the director angles $\phi$ at the upper and lower plates are the same. In other words, the macroscopic polarization is determined by the difference of the director angles at the upper and lower plates. To obtain dynamic behavior of the 
flexoelectric polarization, we consider the director boundary conditions that the director is fixed at the lower plate (i.e., $\mathbf{n}(0)=(1,0,0))$ and weakly restricted at the upper plate. The degree of restriction of the director orientation at the plate surface can be controlled through the surface treatment ${ }^{(17)-(21)}$, such as rubbing surface treatment. This treatment is commonly used in the liquid crystal display industry. The weak director restriction at the upper plate can be taken into account by adding the following surface energy to the Frank energy Eq.(7),

$$
2 F^{\text {surface }}=A\left\{1-\left(\mathbf{n}_{A} \cdot \mathbf{n}_{W}\right)^{2}\right\}
$$

Here, $A$ is the director anchoring strength, $\mathbf{n}_{A}$ the director when there is no torque on the director at the surface, and $\mathbf{n}_{W}$ the actual director at the surface. The above equation shows that the surface energy becomes minimum for $\mathbf{n}_{A} / / \mathbf{n}_{W}$ and maxmum for $\mathbf{n}_{A} \perp \mathbf{n}_{W}$. The value of $A$ is controllable as mentioned above. The director is fixed to $\mathbf{n}_{A}$ in the limit of $A \rightarrow \infty$. The strength $A$ is non-dimensionalized in the same manner as the governing equation, and the non-dimensional director anchoring strength $\mathrm{Ae}\left(=A H^{2} / K\right)$ is also the simulation parameter in addition to the Ericksen number Er. $\mathbf{n}_{A}$ is set to be $(1,0,0)$. The initial condition for the director field is $\mathbf{n}=(1,0,0)$ at entire flow regime including the boundaries.

The material constants, such as viscosities, elasticities, and flexoelectric coefficients, used in the simulation are of $\mathrm{N}$-(p-methoxybenzylidene)-p'-butylaniline (MBBA) ${ }^{(22)-(24)}$ and 4-n-Octyl-4'-cyanobiphenyl (8CB) $)^{(25)-(27)}$ and are shown in Table 1. According to the director behavior in the simple shear flow, MBBA and $8 \mathrm{CB}$ are categorized into the aligning liquid crystal and the tumbling liquid crystal $^{(28)(29)}$. The aligning liquid crystals exhibit the stationary director behavior in the simple shear flows. On the other hand, for the tumbling liquid crystals, the director in the simple shear flows continues rotating.

Table 1 Material constants for MBBA at $298 \mathrm{~K}$ and $8 \mathrm{CB}$ at $310 \mathrm{~K}$
\begin{tabular}{|cc|c|c|}
\hline & & MBBA & $8 \mathrm{CB}$ \\
\hline$\alpha_{1}$ & $(\mathrm{~Pa} \cdot \mathrm{s})$ & $-1.81 \times 10^{-2}$ & $3.82 \times 10^{-2}$ \\
\hline$\alpha_{2}$ & $-1.10 \times 10^{-1}$ & $-5.88 \times 10^{-2}$ \\
\hline$\alpha_{3}$ & $-1.10 \times 10^{-3}$ & $3.05 \times 10^{-3}$ \\
\hline$\alpha_{4}$ & $8.26 \times 10^{-2}$ & $5.2 \times 10^{-2}$ \\
\hline$\alpha_{5}$ & $7.79 \times 10^{-2}$ & $4.73 \times 10^{-2}$ \\
\hline$\alpha_{6}$ & $-3.36 \times 10^{-2}$ & $-8.45 \times 10^{-3}$ \\
\hline$K_{1} \quad(\mathrm{~N})$ & $6.0 \times 10^{-12}$ & $1.15 \times 10^{-11}$ \\
\hline$K_{2}$ & $3.8 \times 10^{-12}$ & $0.52 \times 10^{-11}$ \\
\hline$K_{3}$ & $7.5 \times 10^{-12}$ & $1.13 \times 10^{-11}$ \\
\hline$e_{11}+e_{33}(\mathrm{C} / \mathrm{m})$ & $-5.4 \times 10^{-11}$ & $1.32 \times 10^{-11}$ \\
\hline
\end{tabular}

\section{Simulation results and discussion}

\subsection{Aligning liquid crystal (MBBA)}

As mentioned above, for the aligning liquid crystals, the director stops rotating at the Leslie angle $\phi_{L}\left(=\tan ^{-1} \sqrt{\alpha_{3} / \alpha_{2}}\right)^{(30)(31)}$ under the simple shear flows.

Figure 3 shows the director orientation angle profiles at $t^{*}=5,10$, and 40 , for $\mathrm{Er}=500$ and $\mathrm{Ae}=100$. As time increases, the director angle increases except the angle at the lower plate where the director angle $\phi$ keeps 0 deg because of the director anchoring condition. The increase of the angle at the upper plate is small compared with the angle at the bulk region owing to the weak anchoring ${ }^{(21)}$. The director angle profile stops evoluting when the director angle at the bulk region reaches the Leslie angle $\phi(\approx 5.71 \mathrm{deg}$ for MBBA $)$. The 
director angle at the upper plate becomes different from that at the lower plate because of the difference in the anchoring strength at the lower and upper plates. The macroscopic flexoelectric polarization between the plates can be obtained by integrating the $y$-component of the flexoelectric polarization vector, which is

$$
\left\langle P_{y}^{*}\right\rangle=\int_{0}^{1} P_{y}^{*}\left(y^{*}\right) d y^{*}=\frac{1}{2}\left(n_{y}^{2}(1)-n_{y}^{2}(0)\right)=\frac{1}{2} n_{y}^{2}(1)
$$

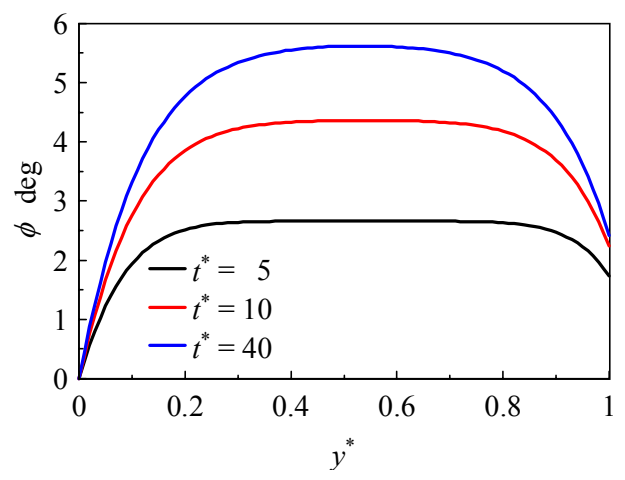

Fig. 3 Director angle profiles for $\mathrm{Er}=500$ and $\mathrm{Ae}=100$

Figure 4 is the transient behavior of the macroscopic flexoelectric polarization $\left\langle P_{y}^{*}\right\rangle$, for $\mathrm{Er}=500$ and $\mathrm{Ae}=100$. The macroscopic polarization increases with time, and finally asymptotically reaches its steady state value. From Eq. (15), it is obvious that the macroscopic flexoelectric polarization is independent of the director profile in the bulk regime. In this work, since the director at the lower plate is fixed, the polarization depends only on the director at the upper plate.

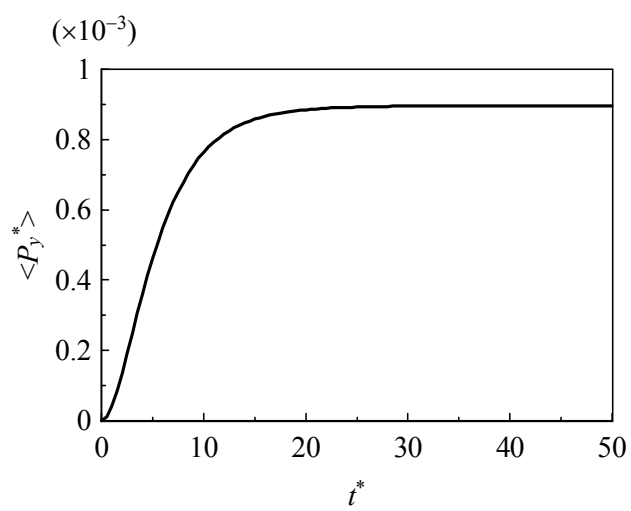

Fig.4 Transient behavior of flexoelectric polarization along $y$-direction for $\mathrm{Er}=500$ and $\mathrm{Ae}=100$

The transient behavior of the director orientation angle at the upper plate is shown in Fig. 5 for $\mathrm{Er}=500$ and $\mathrm{Ae}=100$. It is confirmed that the behavior of the director at the upper plate is quite similar to the behavior of the macroscopic flexoelectric polarization.

Figure 6 shows the steady director profiles for various Ae. The director angle at the upper plate is higher for lower Ae. With increasing Ae, the director profiles become symmetric with respect to the center of the flow channel. The director at the upper plate is determined by the competition between the surface anchoring effect, the shear flow effect, and the long range elasticity effect. In the limit of $\mathrm{Ae} \rightarrow 0$, the surface anchoring effect disappears and the director profile near the upper plate becomes plateau when the Ericksen 
number is sufficiently high.

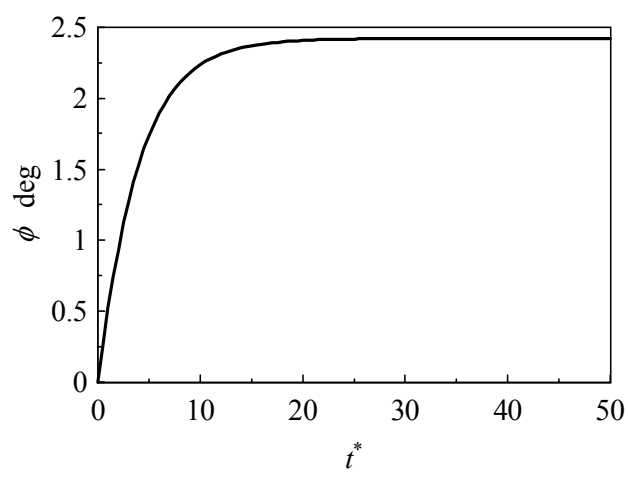

Fig.5 Transient behavior of director angle $\phi$ at upper plate for $\mathrm{Er}=500$ and $\mathrm{Ae}=100$

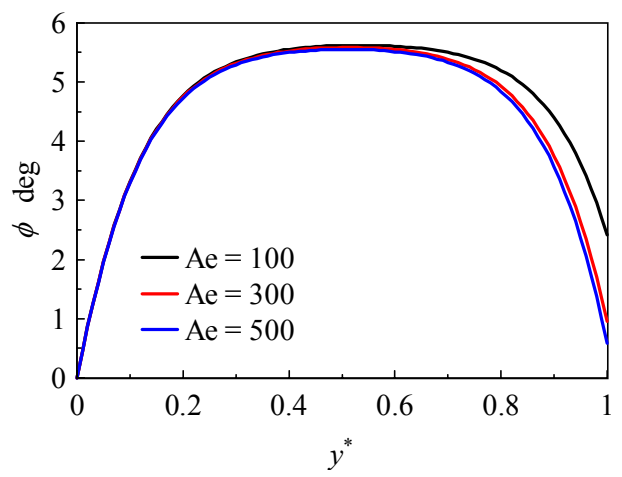

Fig.6 Director angle profiles at steady state

Figure 7 shows the transient behaviors of the macroscopic flexoelectric polarization for $\mathrm{Er}=500$ and $\mathrm{Ae}=100,300$, and 500. The macroscopic polarization is higher for lower Ae, because the director angle at the upper plate is higher for lower Ae as shown in Fig. 6.

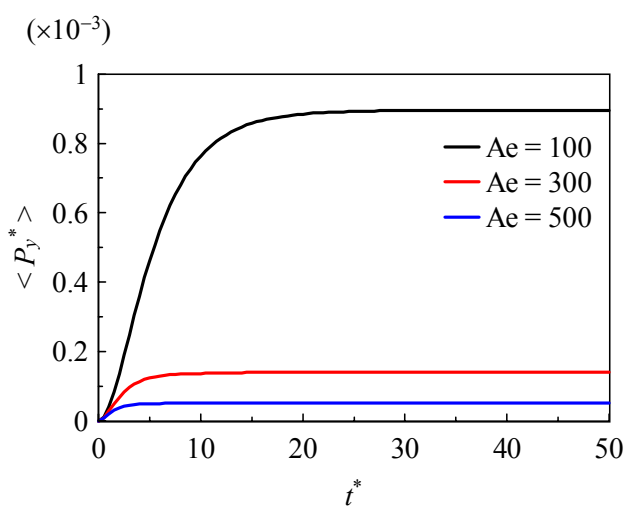

Fig.7 Transient behaviors of the macroscopic flexoelectric polarization for $\mathrm{Er}=500$

Figure 8 shows the effect of Er on flexoelectric polarization. For lower $\operatorname{Er}(\leq 10000)$, the flexoelectric polarization shows sharp increase as Er increase. On the other hand, for higher $\operatorname{Er}(\geq 10000)$, the increase of flexoelectric polarization becomes small. In the limit of $\mathrm{Er} \rightarrow \infty$, the director angle at the upper plate reaches the Leslie angle, and the flexoelectric 
polarization is estimated as:

$$
<P_{y}^{*}>\left.\right|_{E r \rightarrow \infty}=\frac{1}{2} \sin ^{2} \phi_{L}=6.42 \times 10^{-3}
$$

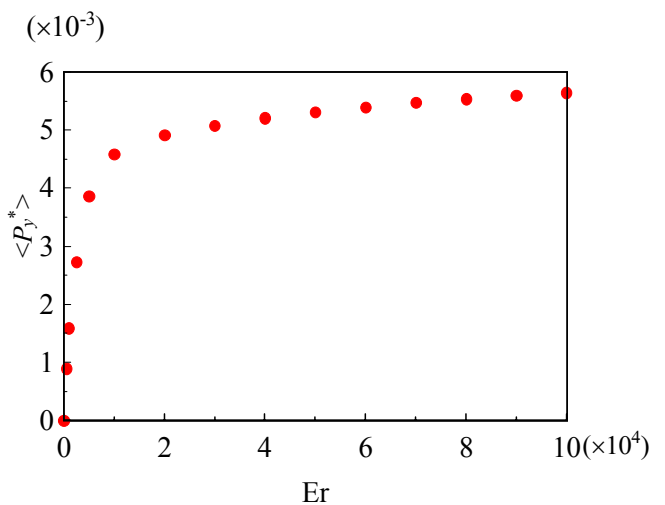

Fig. 8 Effect of Er on flexoelectric polarization for $\mathrm{Ae}=100$

\subsection{Tumbling liquid crystal (8CB)}

It is expected that the behaviors of the macroscopic flexoelectric polarization of the tumbling liquid crystals are drastically different from those of the aligning liquid crystals. For the tumbling liquid crystals ${ }^{(28)(29)}$, one of the Leslie viscosities, $\alpha_{3}$, has opposite sign to $\alpha_{2}$ ( $\alpha_{2}$ is always negative and $\alpha_{3}$ is positive), and thus the Leslie angle cannot be defined. It is known that owing to the sign of $\alpha_{3}$ the flow behavior of the tumbling liquid crystals drastically differs from that of the aligning liquid crystals ${ }^{(32)}$.

Figure 9 shows the director angle profiles at $t^{*}=1,5$, and 20, for $\mathrm{Er}=25$ and $\mathrm{Ae}=100$. The angle decreases as time increases, and the profile reaches the steady state at $t^{*}=20$. The difference of the director angle between the lower and upper plates is found to be about $-0.76 \mathrm{deg}$ in this case. It should be mentioned that the director rotation direction is opposite to that for the aligning liquid crystal (clockwise rotation for the tumbling liquid crystal and counterclockwise rotation for the aligning liquid crystals).

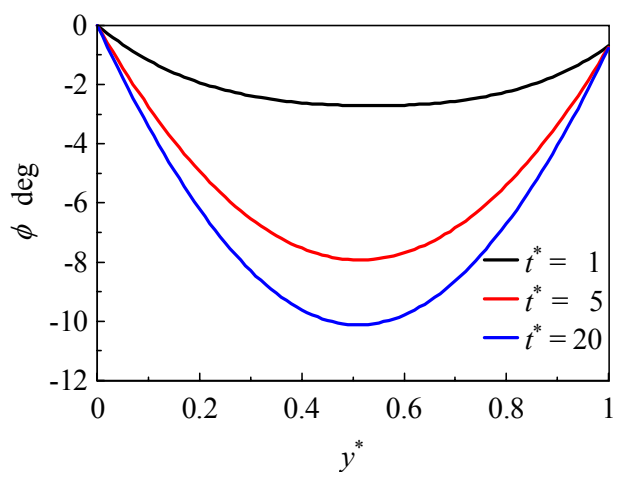

Fig.9 Director angle profiles for $\mathrm{Er}=25$ and $\mathrm{Ae}=100$

To explain this opposite rotation, the effect of shear flow on the director is schematized in Figs. 10(a) and (b) for the aligning liquid crystal and the tumbling liquid crystal, respectively. The arrows in the figure represent the rotation direction of the director. For aligning case, the director always remains in the angle between $+\phi_{L}$ and $-\phi_{L}$ where the director shows counterclockwise rotation. For the tumbling case, on the other hand, the director shows clockwise rotation for any direction. 


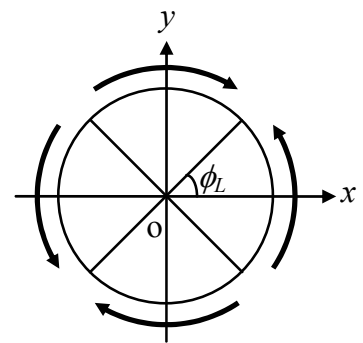

(a)

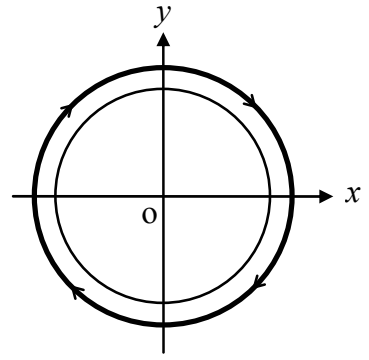

(b)

Fig.10 Effect of shear flow on the director

Figure 11 is the director angle profiles at $t^{*}=5,15$, and 80 , for $\mathrm{Er}=100$ and $\mathrm{Ae}=100$. Because of the high shear rate compared with the case of $\mathrm{Er}=25$ (Fig.9), the director angle is small (the absolute value is large) and its profile is asymmetric. The difference of the director angle between the lower and upper plates reaches about $-365 \mathrm{deg}$ at the steady state.

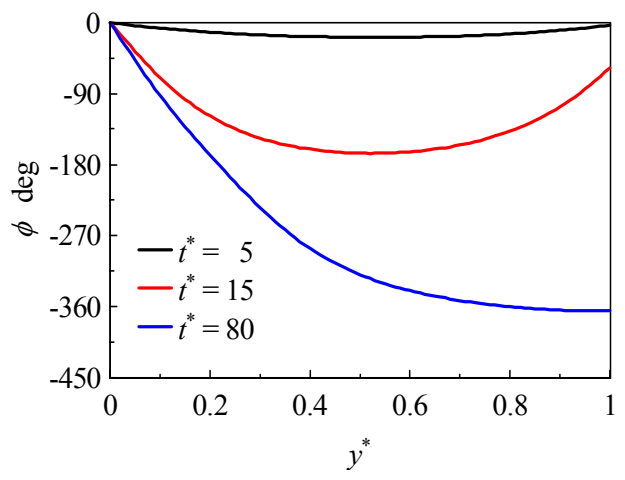

Fig. 11 Director angle profiles for $\mathrm{Er}=100$ and $\mathrm{Ae}=100$

The transient behaviors of the director orientation angle at the upper plate for $\mathrm{Ae}=100$ and $E r=25,50,75,100$, and 125 are shown in Fig.12. Slight decreases of the director angle are found for $\mathrm{Er}=25$ and 50. However, for $\mathrm{Er}=75$, the angles show rapid decrease around $t^{*}=20$ and then reach their steady state angle. For Er=100 and 125, a two-step decrease of the angle is observed before it reaches steady state angle.

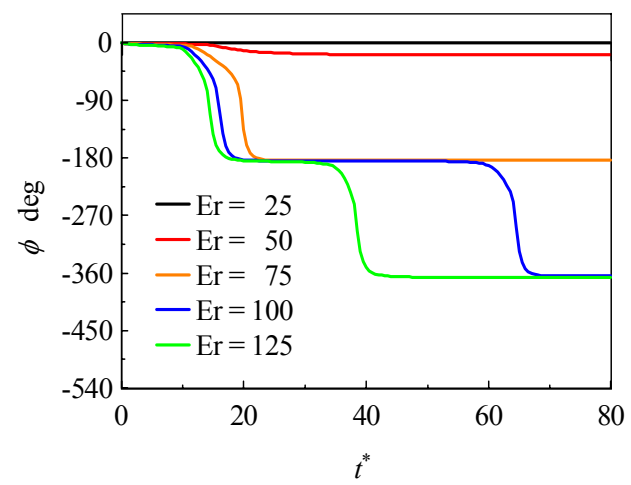

Fig. 12 Transient behaviors of director angle $\phi$ at upper plate for $\mathrm{Ae}=100$ 
To explore the effect of the Ericksen number Er on the director angle at the upper plate, the relation between the angle and Er is shown in Fig.13. The director angle at the upper plate shows discontinuous decrease at $\mathrm{Er} \approx 56.5$ and $\mathrm{Er} \approx 95.5$ and appears every $180 \mathrm{deg}$. When the director is parallel to the shear direction, the viscous torque on the director is the maximum which overcomes the anchoring torque. Thus, there is no director angle around $\phi=180(n+1 / 2) \operatorname{deg}(n=0,-1,-2, \cdots)$.

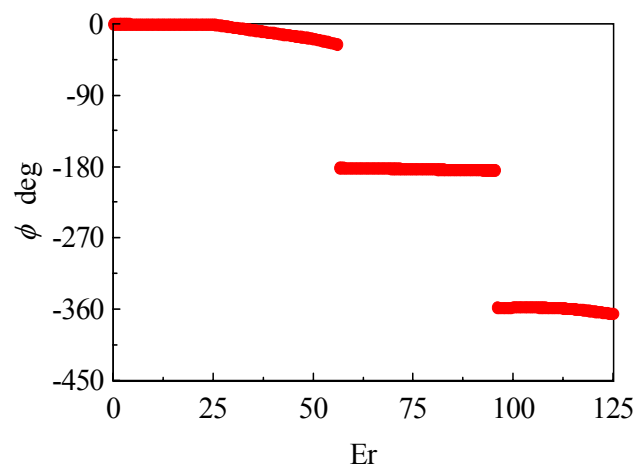

Fig.13 Effect of Er on the director angle at the upper plate for $\mathrm{Ae}=100$

On the other hand, the effect of Ae on the director angle at the upper plate is shown in Fig. 14. The discontinuous increase is observed with increasing Ae. The effect of Ae on the director angle at the upper plate is opposite to that of Er, since the anchoring torque simply increases with increasing Ae.

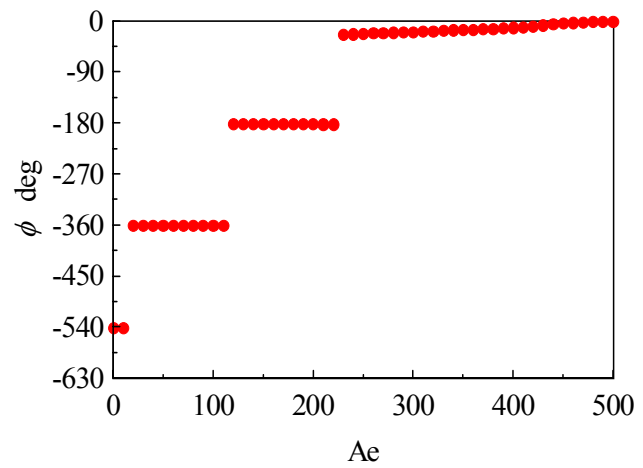

Fig.14 Effect of Ae on the director angle at the upper plate for $\mathrm{Er}=100$

Figure 15 shows the macroscopic flexoelectric polarization as a function of time, for $\mathrm{Ae}=100$ and $\mathrm{Er}=50,100$, and 125. For $\mathrm{Er}=50$, the polarization slightly increases with time, and then reaches the steady value. As mentioned above, the macroscopic flexoelectric polarization is the function of the director at the upper plate, and thus the polarization profile is quite similar to the profile of the upper plate angle shown in Fig.12. However, for $\mathrm{Er}=100$ and 125 , the polarization profile is different from the angle profile. At the time that the angle decreases steeply, the macroscopic flexoelectric polarization shows sharp peaks, since the polarization becomes the maximum when the director at the upper plate is perpendicular to the director at the lower plate. 


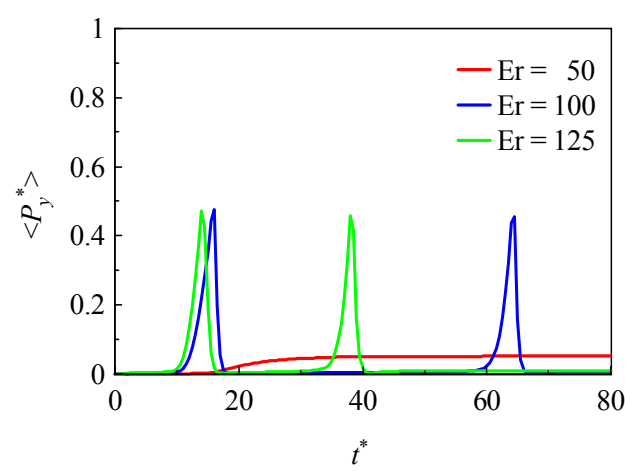

Fig. 15 Flexoelectric polarization as a function of time for $\mathrm{Ae}=100$

\section{Conclusion}

As a fundamental research of the development of new liquid crystalline devices, the flow induced flexoelectric effect is numerically analyzed, using the Leslie-Ericksen continuum theory. The results obtained in this study are as follows.

(1) The flexoelectric polarization for the aligning liquid crystal increases monotonically to reach a steady state value. On the other hand, it shows peaks for tumbling liquid crystal, and the number of peaks depends on Er.

(2) The peak value of the polarization for the tumbling liquid crystal is considerably larger than the steady value for the aligning liquid crystal.

(3) The director angle at the upper plate shows the discontinuous changing with increase of Ae or Er and appears every $180 \mathrm{deg}$ for the tumbling liquid crystal at higher Er and lower Ae.

(4) The effect of Ae on the director angle at the upper plate is opposite to that of Er.

\section{References}

(1) Chandrasekhar, S., Liquid Crystals 2nd Ed., (1992), Cambridge University Press.

(2) de Gennes, P.G., The Physics of Liquid Crystals, 1974, , Clarrendon, Oxford.

(3) Chono, S. and Tsuji, T., Development of Micro-Actuators Driven by Liquid Crystals (1st Report, Generation of Flow and Its Mechanism), Transactions of the Japan Society of Mechanical Engineers, Series B, Vol.72, No.715 (2006), pp.96-101.

(4) Liu, C. et al., Development of Micro-Actuators Driven by Liquid Crystals (2nd Report, Effect of Various Parameters), Transactions of the Japan Society of Mechanical Engineers, Series B, Vol.72, No.721 (2006), pp.2235-2241.

(5) Meyer, R.B., Piezoelectric Effects in Liquid Crystals, Phys. Rev. Lett., Vol.22(1969), pp.918-921.

(6) de Gennes, P.G., The Physics of Liquid Crystals, 1974, P.135, Clarrendon, Oxford.

(7) Valenti, B. et al., Flexoelectricity in the Hybrid AlignedNematic Cell, Mol. Cryst. Liq. Cryst., Vol.146 (1987), pp. 307-320.

(8) Madhusudana, N. V. and Durand, G. , Linear flexo-electro-optic effect in a hybrid aligned nematic liquid crystal cell, J. Phys. Lett., Vol. 46(1985), pp.L-195-L-200.

(9) Stelzer, J. et al., Flexoelectric effects in liquid crystals formed by pear-shaped molecules. A computer simulation study, Chem. Phys. Lett., Vol.299 (1999), pp.9-16.

(10) Billeter, J.L. and Pelcovits, R.A., Molecular shape and flexoelectricity, Liq.Cryst., Vol.27, No.9(2000), pp.1151-1160. 
(11) Jenkins, J.T. Flows of nematic liquid crystals, Ann. Rev. Fluid Mech., Vol.10(1978), pp. 197-219.

(12) Leslie, F.M., Theory of Flow Phenomena in Liquid Crystals, Adv. Liq. Cryst., Vol.4(1979), pp.1-81.

(13) Chono, S., Tsuji, T. and Denn, M.M., Numerical Simulation of Planar Contraction Flow of Nematic Liquid Crystals, Trans. Japan Soc. Mech. Eng. B, Vol. 60(1994), pp.1944-1950.

(14) Leslie, F.M., Some Constitutive Equations for Liquid Crystals, Arch. Ration.Mech.Anal., Vol.28, No.4 (1968), pp.265-283.

(15) Ericksen, J.L., Anisotropic fluids, Arch. Ration. Mech. Anal. 4, Vol. 28(1960), pp.231.

(16) Ericksen, J.L., Conservation Laws for Liquid Crystals, Trans. Soc. Rheol., Vol. 5(1961), pp. 23-24.

(17) Warenghem, M., Grandjean-Cano Texture under Weak Anchoring Conditions in Cholesteric Cells, Mol. Cryst. Liq. Cryst., Vol. 220(1992), pp.39-51.

(18) Sato, Y. et al., Relationship between Rubbing Strength and Surface Anchoring of Nematic Liquid Crystal, Appl. Phys. Vol. 31(1992), pp.L579-L581.

(19) Yokoyama, H. and van Sprang, H. A., A novel method for determining the anchoring energy function at a nematic liquid crystal-wall interface from director distortions at high fields, J. Appl. Phys, Vol. 57(1985), pp.4520-4526.

(20) Yokoyama, H. et al., Temperature dependence of the anchoring strength at a nematic liquid crystal-evaporated SiO interface, J. Appl. Phys, Vol. 61(1987), pp.4501-4518.

(21) Yokoyama, H., Surface Anchoring of Nematic Liquid crystals, Mol. Cryst. Liq. Cryst., Vol.165 (1988), pp.265-316.

(22) Takahashi, T. et al., Novel Measurement Method for Flexoelectric Cofficients of Nematic Liquid Crystals, Jpn. J.Appl. Phys, Vol.37, No. 4A(1998), pp.1865-1869.

(23) Kneppe, H. et al., Rotational viscosity $\gamma_{1}$ of nematic liquid crystals, J. Chem. Phys, Vol.77(1982), pp.3203-3208.

(24) Haller, I., Elastic Constants of the Nematic Liquid Crystalline Phase of pMethoxybenzylidene - p-n-Butylaniline (MBBA), J. Chem. Phys., Vol.57(1972), pp.1400-1405.

(25) Karat, P.P., and Madhusudana, N.V., Elasticity and orientational order in some 4'-n-alkyl-4-cyanobiphenyls, Mol. Cryst. Liq. Cryst., Vol.40(1977), pp.239-245.

(26) Kneppe, H. et el., A comparative study of the viscosity coefficients of some nematic liquid crystals, Ber.Bunsenges.Phys.Chem, Vol.85(1981), pp.784-789.

(27) Maheswara, P. R. et al., Experimental determination of the flexoelectric coefficients of some nematic liquid crystals, Liq. Cryst., Vol. 14, No.2(1993), pp.483-496.

(28) Pieranski, P. and Guyon, E., Two Shear-Flow Regimes in Nematic p-nHexyloxybenzilidene-p'-aminobenzonitrile, Phys. Rev. Lett., Vol. 32(1974), pp.924-926.

(29) Cladis, P. E. and S. Torza, S., Stability of Nematic Liquid Crystals in Couette Flow, Phys. Rev. Lett., Vol.35 (1975), pp.1283-1286.

(30) Gahwiller, Ch., Direct determination of the five independent viscosity coefficients of nematic liquid crystals, Mol. Cryst. Liq. Cryst., Vol.20(1973), pp.301-318.

(31) Gahwiller, Ch., Temperature Dependence of Flow Alignment in Nematic Liquid Crystals, Phys. Rev. Lett., Vol. 28, No.24(1972), pp.1554-1556.

(32) Carlsson, T., and Skarp, K., Observation of tumbling instability in torsional shear flow of a nematic liquid crystal with $\alpha_{3}>0$, Liq. Cryst., Vol. 1(1986), pp.455-471. 